\title{
Una aproximación a la conciliación familiar y \\ laboral en la Unión Europea
}

\author{
Llorent-Bedmar, Vicente \\ Facultad de Ciencias de la Educación, Sevilla, España \\ llorent@us.es \\ Cobano-Delgado Palma, Verónica \\ Facultad de Ciencias de la Educación, Sevilla, España \\ cobano@us.es
}

\section{Resumen}

La unidad familiar ha sufrido una considerable evolución en las últimas décadas, especialmente en las grandes ciudades, donde se han operado importantes cambios en las condiciones laborales, en los horarios, en las distancias... Sin pretender hacer una exhaustiva descripción, nos aproximamos a las políticas de apoyo a las familias en el marco de la Unión Europea, resaltando aquellas cuestiones que estimamos más interesantes.

Tras referirnos a las políticas de conciliación familiar-laboral que se llevan a cabo en los países de la UE, nos centramos en los permisos de maternidad y paternidad, y en las ayudas por nacimiento que se conceden. Hemos podido comprobar la heterogeneidad de las políticas de apoyo a las familias que se están desarrollando en los distintos Estados miembros de la UE en función del modelo de bienestar seguido en cada país. Dichas políticas pretenden disminuir algunas de las dificultades económicas y laborales que se derivan fundamentalmente del nacimiento de los hijos. En líneas generales, a tal efecto en los países estudiados se pretende realizar un reparto equitativo de las responsabilidades y prestar una mayor atención a determinadas situaciones familiares.

\section{Abstract}

The family has changed considerably in recent decades, especially in large cities, where there have been important changes in working conditions, in schedules, in distances ... We do not carry out an exhaustive description, but a panoramic vision of the policies of support to families in the European Union, highlighting those aspects that we consider most interesting.

After referring to the family-labor conciliation policies of the EU countries, we focus on maternity and paternity leave, and on the grants that are granted for the birth of children. We verified that there are different policies of support to families in the countries of the EU according to the welfare model of each country. These policies aim to reduce economic and labor difficulties that occur after the birth of children. The EU countries aim to make an equal distribution of responsibilities and give greater attention to certain family situations.

Palabras clave: familia, infancia, conciliación, ayudas, bienestar de la infancia.

Keywords: family, childhood, conciliation, aid, child protection.

\section{INTRODUCCIÓN}

Alcanzar una conciliación familiar-laboral de calidad es un aspecto de indudable importancia para contribuir, entre otros, al logro de los siguientes objetivos: A. Favorecer el empleo (especialmente entre las mujeres); B. Promover el desarrollo de la infancia; C. Fomentar la igualdad de género (Davies, 2013). 
Centrándonos en el primer y tercer objetivo de las políticas de conciliación, fomento del empleo femenino y de la igualdad de género, autoras como González (2014), destacan que, a pesar de los esfuerzos realizados desde la Unión Europea (UE), sigue presente un sesgo masculino que conlleva de manera inexorable a que la mujer siga asumiendo en mayor medida las tareas domésticas y el cuidado de los hijos. Motivo por el que la elección de trabajos a tiempo parcial por parte de las mujeres ha sido la práctica más generalizada en los Estados miembro de la UE, especialmente cuando sus hijos inician el período escolar (McColgan, 2015).

No obstante, tan sólo el $66 \%$ de las mujeres con hijos menores de 14 años se encontraban en 2016 activas en el mercado laboral. Aunque estas tasas de empleo difieren de manera considerable entre un país y otro de la UE, son especialmente elevadas en Suecia y Dinamarca (82/83\%), y mucho más bajas en países como Italia, Grecia, Hungría, República Checa y España (60\%) (OCDE, 2016a).

Lograr una conciliación familiar-laboral de calidad puede facilitarse en gran medida mediante políticas de apoyo a las familias. Con el presente trabajo ${ }^{1}$ pretendemos aproximarnos a las políticas de apoyo a las familias que se desarrollan en el marco de la UE, resaltando los aspectos que estimamos de especial interés. Concretamente, nos centraremos en los permisos parentales y las prestaciones por nacimiento, que constituyen un exponente de la importancia cada país le otorga a la atención y protección de la infancia.

Con respecto a los permisos de paternidad, investigaciones e informes de índole internacional concluyen una desigual distribución de estos permisos entre madres y padres, siendo las primeras las que optan por este permiso prácticamente en su totalidad (McColgan, 2015). Este hecho afeta indudablemente al empleo femenino, limitando la participación de la mujer en el mercado laboral remunerado, incluso teniendo que renunciar a su desarrollo profesional (López, Acereda, Signes y Amado, 2015).

\section{ATENCIÓN A LA INFANCIA EN PAÍSES DE LA UNIÓN EUROPEA}

Según datos de Eurostat la participación en la atención y educación de la primera infancia ha ido aumentando de manera continua desde 2002. Actualmente, el porcentaje medio de escolarización de niños de 4 años de edad es del 94,8\%, aproximándose casi por completo al índice de escolarización que se propuso en la Estrategia 2020 (alcanzar una tasa de escolarización de, al menos, el 95\%) (Eurostat, 2017).

En Europa, los servicios que actualmente se prestan para la atención a la primera infancia se caracterizan por ser heterogéneos y dinámicos. Son considerables las diferencias que encontramos entre países, tanto en la educación formal como en la no formal. En Europa constatamos la coexistencia de dos modelos organizativos distintos en la educación formal: un modelo de etapa única (normalmente destinado a niños de 0 a 6 años) y un modelo de dos etapas diferenciadas (normalmente de 0 a 3 y de 6 a 6 años). La mayoría de los países de la UE siguen el modelo diferenciado, donde incluso el organismo encargado de formular las políticas difiere para cada etapa. El primer ciclo de Educación Infantil se orienta más bien hacia las políticas de asuntos sociales y de apoyo a las familias, mientras que el segundo lo hace hacia el ámbito educativo (Eurydice, 2012).

En el ámbito de las políticas familiares existan diversas directivas promulgadas por la UE, si bien cada Estado miembro tiene competencias al respecto. Con mayor o menor acierto, todos los países están adoptando medidas políticas tendentes a favorecer la conciliación entre la actividad profesional y la familiar. Estas políticas familiares han de fomentar el crecimiento. Es a partir de 1989 cuando con el Informe sobre la comunicación de la Comisión de la UE sobre políticas familiares se promueve abordar desde el ámbito legislativo políticas legislativas en pro de esta conciliación ${ }^{2}$. En ese mismo año se crea el Observatorio Europeo de las Políticas Familiares Nacionales al que se le encarga el seguimiento de las políticas familiares y del estudio de las familias en los distintos países de la UE.

\footnotetext{
${ }^{1}$ En el marco del proyecto I+D con referencia EDU 2016-78134-R, financiado por la Agencia Estatal de Investigación (AEI) y el Fondo Europeo de Desarrollo Regional (FEDER).

${ }^{2}$ Informe sobre la conciliación de la vida profesional, familiar y privada (2003/2129 (INI)), Comisión de derechos de la mujer e igualdad de oportunidades. 18 de diciembre de 2003. p. 10.
} 
Considerando la necesidad de desarrollar medidas de intervención que faciliten la conciliación de las responsabilidades familiares y laborales, tanto para el hombre como para la mujer, así como la educación de los hijos -especialmente, la primera infancia-, en 1992 se encomendó a los Estados miembro adoptar y fomentar iniciativas orientadas a conciliar la vida profesional, familiar y educativa que supone tener un hijo (artículo primero de la Recomendación del Consejo de 31 de marzo sobre el cuidado de los niños y niñas ${ }^{3}$ ).

En 1992 el Consejo de la UE aprobó la Directiva 92/85/CEE, relativa a la aplicación de medidas para promover la mejora de la seguridad y de la salud en el trabajo de la trabajadora embarazada, que haya dado a luz o en el periodo de lactancia. En su artículo ocho se indicaba que, con arreglo a las legislaciones y prácticas nacionales, los Estados miembros deben tomar las medidas que consideren para que toda mujer trabajadora embarazada, que dé a luz o en periodo de lactancia ${ }^{4}$ disfrute de un permiso de maternidad mínimo establecido en 14 semanas, en las que se deben incluir las dos semanas anteriores y dos semanas posteriores al parto (art. 8). En esta misma línea, las Naciones Unidas proclamaron 1994 como el Año Internacional de la Familia, cuyas acciones y objetivos contribuyeron significativamente a la toma de conciencia sobre la importancia de la institución familiar.

Así pues, podemos afirmar que desde 1990 hasta la actualidad, en el ámbito de la UE se han aprobado medidas legislativas de importancia en pro de la conciliación familiar y laboral. Así, el 29 de junio de 2000, el Consejo Europeo adoptó una resolución relativa a la participación equilibrada de hombres y mujeres en la actividad profesional y en la vida familiar ${ }^{5}$, alentando a los Estados Miembros a armonizar los horarios escolares y laborales. Asimismo, en 2008, se publicó una nueva propuesta que modificaba a esta directiva, extendiendo este período de 14 a 18 semanas. Finalmente, en 2010 el Parlamento Europeo presentó un período de paternidad intransferible de, al menos, 2 semanas (Eurofound, 2015).

\subsection{Permisos de paternidad y pagos por nacimiento}

A continuación, analizamos los permisos de maternidad y paternidad que los distintos países de la UE ofrecen actualmente para facilitar una mejor conciliación de la vida familiar y laboral, atendiendo a la duración (obligatoria y no obligatoria), porcentaje del sueldo que perciben ambos progenitores y la transferencia o no del permiso.

\footnotetext{
${ }^{3}$ Recomendación del Consejo de 31 de marzo de 1992 sobre el cuidado de los niños y las niñas (92/241/CEE).

${ }^{4}$ Según el artículo 2 de la Directiva 92/85/CEE se entiende:

a) trabajadora embarazada: cualquier trabajadora embarazada que comunique su estado al empresario, con arreglo a las legislaciones y/o prácticas nacionales;

b) trabajadora que ha dado a luz: cualquier trabajador que haya dado a luz en el sentido de las legislaciones y/o prácticas nacionales, que comunique su estado al empresario, con arreglo a dichas legislaciones y/o prácticas nacionales;

c) trabajadora en periodo de lactancia: cualquier trabajadora en periodo de lactancia en el sentido de las legislaciones y/o prácticas nacionales, que comunique su estado al empresario, con arreglo a dichas legislaciones y/o prácticas nacionales.

${ }^{5}$ Diario Oficial $n^{\circ} \mathrm{C} 218$ de 31/07/2000, p. 0005.
} 
Tabla 1. Permisos de maternidad y paternidad en países de la UE.

\begin{tabular}{|c|c|c|c|c|c|}
\hline & PERMISO DE MATERNIDAD & $\begin{array}{l}\% \text { DEL } \\
\text { SUELDO }\end{array}$ & $\begin{array}{l}\text { PERMISO DE } \\
\text { PATERNIDAD }\end{array}$ & $\begin{array}{l}\% \text { DEL } \\
\text { SUELDO }\end{array}$ & TRANSFERIBLE \\
\hline ALEMANIA & $\begin{array}{l}\text { - Permiso prenatal: } 6 \text { semanas. } \\
\text { - Permiso de posparto: } 8 \text { semanas } \\
\text { (obligatorias) }\end{array}$ & 100 & - & - & No \\
\hline AUSTRIA & $\begin{array}{l}\text { - Permiso prenatal: } 8 \text { semanas. } \\
\text { - Permiso de posparto: } 8 \text { semanas } \\
\text { (ambos obligatorios) }\end{array}$ & 100 & - & - & No \\
\hline BÉLGICA & $\begin{array}{l}\text { - Permiso prenatal: } 6 \text { semanas. } \\
\text { - Permiso de posparto: } 9 \text { semanas } \\
\text { (obligatorias) }\end{array}$ & 100 & $\begin{array}{l}2 \text { semanas ( } 3 \\
\text { días obligato- } \\
\text { rios) }\end{array}$ & 82 & $\begin{array}{l}\text { Sólo en caso de } \\
\text { muerte materna u } \\
\text { hospitalización }\end{array}$ \\
\hline BULGARIA & $\begin{array}{l}\text {-Permiso prenatal: } 6 \text { semanas } \\
\text { (obligatorias). } \\
\text {-Permiso postnatal: } 52 \text { semanas }\end{array}$ & 90 & $\begin{array}{c}2 \text { semanas (no } \\
\text { obligatorias) }\end{array}$ & 90 & $\begin{array}{l}\text { Sí, después de que } \\
\text { el niño tenga } \\
\text { seis meses }\end{array}$ \\
\hline CHIPRE & $\begin{array}{l}\text {-Permiso prenatal: } 9 \text { semanas (2 } \\
\text { semanas obligatorias). } \\
\text {-Permiso posnatal: } 9 \text { semanas }\end{array}$ & 72 & - & - & No \\
\hline CROACIA & $\begin{array}{l}\text {-Permiso prenatal: } 8 \text { semanas } \\
\text { (obligatorias). } \\
\text {-Permiso posnatal: } 26 \text { semanas } \\
\text { (10 obligatorias) }\end{array}$ & 100 & - & - & $\begin{array}{l}\text { Sí, después de } \\
\text { las primeras } 14 \\
\text { semanas }\end{array}$ \\
\hline DINAMARCA & $\begin{array}{l}\text {-Permiso prenatal: } 4 \text { semanas. } \\
\text {-Permiso postnatal: } 14 \text { semanas } \\
\text { ( } 2 \text { obligatorias) }\end{array}$ & 100 & $\begin{array}{c}2 \text { semanas (no } \\
\text { obligatorias) }\end{array}$ & 100 & $\begin{array}{l}\text { Sólo en caso de } \\
\text { enfermedad por } \\
\text { parte de la madre }\end{array}$ \\
\hline ESLOVAQUIA & $\begin{array}{l}\text {-Permiso prenatal: } 8 \text { semanas } \\
\text { (obligatorias). } \\
\text {-Permiso posnatal: } 26 \text { semanas } \\
\text { (6 obligatorias) }\end{array}$ & $65 \%$ & - & - & No \\
\hline ESLOVENIA & $\begin{array}{l}\text {-Permiso prenatal: } 4 \text { semanas } \\
\text { (2 obligatorias). } \\
\text {-Permiso posnatal: } 11 \text { semanas }\end{array}$ & 100 & $\begin{array}{c}10 \text { semanas } \\
\text { (no obligatorias) }\end{array}$ & $\begin{array}{l}20 \text { días se } \\
\text { paga el } \\
90 \% \text { del } \\
\text { sueldo y los } \\
\text { próximos } \\
50 \text { días se } \\
\text { pagan a } \\
\text { través de } \\
\text { las cotiza- } \\
\text { ciones a la } \\
\text { seguridad } \\
\text { social. }\end{array}$ & $\begin{array}{c}\text { Sólo en caso de } \\
\text { fallecimiento de la } \\
\text { madre o } \\
\text { enfermedad }\end{array}$ \\
\hline ESPAÑA & $\begin{array}{l}\text {-Permiso prenatal: } 10 \text { semanas. } \\
\text {-Permiso posnatal: } 6 \text { semanas } \\
\text { (obligatorias). }\end{array}$ & 100 & $\begin{array}{c}2 \text { semanas (no } \\
\text { obligatorias) }\end{array}$ & 100 & $\begin{array}{c}\text { Sí, después de } \\
\text { las primeras seis } \\
\text { semanas o en caso } \\
\text { de fallecimiento de } \\
\text { la madre. }\end{array}$ \\
\hline ESTONIA & $\begin{array}{l}\text {-Permiso prenatal: } 10 \text { semanas. } \\
\text {-Permiso posnatal: } 10 \text { semanas } \\
\text { (obligatorias). }\end{array}$ & 100 & $\begin{array}{c}2 \text { semanas (no } \\
\text { obligatorias) }\end{array}$ & 100 & No \\
\hline
\end{tabular}




\begin{tabular}{|c|c|c|c|c|c|}
\hline FINLANDIA & $\begin{array}{l}\text {-Permiso prenatal: } 8 \text { semanas } \\
\text { ( } 2 \text { obligatorias). } \\
\text {-Permiso posnatal: } 9 \text { semanas } \\
\text { ( } 2 \text { obligatorias). }\end{array}$ & $\begin{array}{l}\text { Primeros } \\
56 \text { días } \\
\text { el } 90 \% \text { el } \\
\text { resto el } \\
70 \% .\end{array}$ & $\begin{array}{l}9 \text { semanas (no } \\
\text { obligatorias) }\end{array}$ & Variable & No \\
\hline FRANCIA & $\begin{array}{l}\text {-Permiso prenatal: } 2 \text { semanas } \\
\text { (obligatorias). } \\
\text {-Permiso posnatal: } 14 \text { semanas. }\end{array}$ & 100 & $\begin{array}{c}2 \text { semanas (no } \\
\text { obligatorias) }\end{array}$ & 100 & No \\
\hline GRECIA & $\begin{array}{l}\text {-Permiso prenatal: } 8 \text { semanas } \\
\text { (obligatorias). } \\
\text {-Permiso posnatal: } 12 \text { semanas } \\
\text { (obligatorias) }\end{array}$ & 100 & 2 días & 100 & No \\
\hline HUNGRÍA & $\begin{array}{l}\text {-Permiso prenatal: } 4 \text { semanas. } \\
\text {-Permiso posnatal: } 20 \text { semanas } \\
\text { ( } 2 \text { obligatorias). }\end{array}$ & 70 & $\begin{array}{c}1 \text { semana (no } \\
\text { obligatoria) }\end{array}$ & 100 & No \\
\hline IRLANDA & $\begin{array}{l}\text {-Permiso prenatal: } 2 \text { semanas } \\
\text { (obligatorias). } \\
\text {-Permiso posnatal: } 40 \text { semanas } \\
\text { (4 obligatorias). }\end{array}$ & $\begin{array}{c}\text { Tarifa } \\
\text { semanal } \\
(230 €) \text { du- } \\
\text { rante } 26 \\
\text { semanas. } \\
\text { Las res- } \\
\text { tantes } 16 \\
\text { semanas } \\
\text { sin sueldo }\end{array}$ & $\begin{array}{l}2 \text { semanas } \\
\text { (no obligatorias) }\end{array}$ & Variable & $\begin{array}{l}\text { Sólo en caso de } \\
\text { fallecimiento de la } \\
\text { madre }\end{array}$ \\
\hline ITALIA & $\begin{array}{l}\text {-Permiso prenatal: } 4 \text { semanas } \\
\text { (obligatorias). } \\
\text {-Permiso posnatal: } 8 \text { semanas }\end{array}$ & 100 & 2 días & 100 & $\begin{array}{l}\text { Sólo en caso de } \\
\text { fallecimiento de la } \\
\text { madre, } \\
\text { enfermedad o } \\
\text { abandono. }\end{array}$ \\
\hline LETONIA & $\begin{array}{l}\text {-Permiso prenatal: } 8 \text { semanas } \\
\text { ( } 2 \text { obligatorias). } \\
\text {-Permiso posnatal: } 8 \text { semanas } \\
\text { ( } 2 \text { obligatorias). }\end{array}$ & 80 & 1,4 semanas & 80 & No \\
\hline LITUANIA & $\begin{array}{l}\text {-Permiso prenatal: } 10 \text { semanas. } \\
\text {-Permiso posnatal: } 8 \text { semanas. }\end{array}$ & 100 & 4 semanas & 100 & No \\
\hline LUXEMBURGO & $\begin{array}{l}\text {-Permiso prenatal: } 8 \text { semanas } \\
\text { (obligatorias) } \\
\text { - Permiso de posparto: } 8 \text { semanas } \\
\text { (obligatorias) }\end{array}$ & 100 & - & - & No \\
\hline MALTA & $\begin{array}{l}\text {-Permiso prenatal: } 8 \text { semanas. } \\
\text {-Permiso posnatal: } 10 \text { semanas } \\
\text { ( } 6 \text { obligatorias). }\end{array}$ & $\begin{array}{l}14 \text { se- } \\
\text { manas el } \\
100 \%\end{array}$ & 2 días & 100 & No \\
\hline PAÍSES BAJOS & $\begin{array}{l}\text {-Permiso prenatal: } 6 \text { semanas } \\
\text { (4 obligatorias). } \\
\text {-Permiso posnatal: } 10 \text { semanas } \\
\text { (obligatorias). }\end{array}$ & 100 & 2 días & 100 & $\begin{array}{l}\text { Solo en caso de } \\
\text { fallecimiento de la } \\
\text { madre }\end{array}$ \\
\hline POLONIA & $\begin{array}{l}\text {-Permiso prenatal: } 6 \text { semanas } \\
\text { (obligatorias). } \\
\text {-Permiso posnatal: } 14 \text { semanas } \\
\text { (8 obligatorias). }\end{array}$ & Variable & 2 días & 100 & $\begin{array}{l}\text { Sí, después de las } \\
\text { primeras } \\
14 \text { semanas }\end{array}$ \\
\hline PORTUGAL & $\begin{array}{l}\text {-Permiso prenatal: } 4 \text { semanas. } \\
\text {-Permiso posnatal: } 6 \text { semanas } \\
\text { (obligatorias). }\end{array}$ & 100 & $\begin{array}{l}5 \text { días (3 obli- } \\
\text { gatorios) }\end{array}$ & 100 & $\begin{array}{l}\text { Sí, después de las } \\
\text { primeras } \\
6 \text { semanas tras } \\
\text { el nacimiento }\end{array}$ \\
\hline
\end{tabular}




\begin{tabular}{|c|c|c|c|c|c|}
\hline REINO UNIDO & $\begin{array}{l}\text {-Permiso prenatal: } 11 \text { semanas } \\
\text {-Permiso posnatal: } 41 \text { semanas } \\
\text { ( } 2 \text { obligatorias). }\end{array}$ & $\begin{array}{l}\text { Las pri- } \\
\text { meras } 6 \\
\text { semanas } \\
\text { el } 90 \% \text {, } \\
33 \text { sema- } \\
\text { nas } 165 € \\
\text { o el } 90 \% \\
\text { de los } \\
\text { ingresos } \\
\text { brutos } \\
\text { semana- } \\
\text { les. Las } \\
\text { ultimas } 13 \\
\text { no remu- } \\
\text { neradas. }\end{array}$ & 2 semanas & variable & $\begin{array}{l}\text { Sí, distinto del } \\
\text { período obligatorio } \\
\text { de dos semanas }\end{array}$ \\
\hline $\begin{array}{c}\text { REPÚBLICA } \\
\text { CHECA }\end{array}$ & $\begin{array}{l}\text {-Permiso prenatal: } 8 \text { semanas } \\
\text { (obligatorias). } \\
\text {-Permiso posnatal: } 20 \text { semanas } \\
\text { (6 obligatorias). }\end{array}$ & 70 & - & - & No \\
\hline RUMANÍA & $\begin{array}{l}\text {-Permiso prenatal: } 9 \text { semanas. } \\
\text {-Permiso posnatal: } 9 \text { semanas } \\
\text { (6 obligatorias). }\end{array}$ & $85 \%$ & 1 semana & variable & No \\
\hline SUECIA & $\begin{array}{l}\text {-Permiso prenatal: } 7 \text { semanas } \\
\text { ( } 2 \text { obligatorias). } \\
\text {-Permiso posnatal: } 7 \text { semanas. }\end{array}$ & Opcional & 2 semanas & $80 \%$ & No \\
\hline
\end{tabular}

Fuente: Elaboración propia a partir de European Parlament (2016) y McColgan (2015).

Como podemos observar en la tabla 1, los permisos de maternidad y paternidad difieren de unos países a otros, aunque desde la UE se vayan marcando las posibles líneas de actuación y unos derechos mínimos a respetar, cada Estado tiene competencia explícita en esta materia.

Respecto al permiso de maternidad, Bulgaria (58 semanas), Reino Unido (52 semanas) e Irlanda (42 semanas) son los países que ofrecen un permiso de mayor duración (pre y posnatal). Si nos referimos específicamente al permiso obligatorio, Grecia (20 semanas) es el país que proporcionan el permiso obligatorio más extenso.

Casi todos los países de la UE ofrecen permisos de maternidad de una duración de, al menos, tres meses, en consonancia con la actual directiva de la UE que estipula que las madres deben tener acceso, al menos, a 14 semanas de permiso (OCDE, 2017).

Respecto al permiso de paternidad, llama especialmente la atención que tan solo dos países -Portugal y Bélgica- tengan estipulado un permiso de paternidad obligatorio (mayor en el primero). El resto de países les conceden a los padres un permiso opcional, que oscila desde las 10 semanas en Eslovenia y las 9 en Finlandia, hasta tan sólo 1 día en Rumanía y Hungría. Finalmente, destacamos como en muchos países de la UE no se contempla el permiso paternal, como son los casos de Dinamarca, República Checa, Luxemburgo, Eslovaquia, Croacia, Chipre, Austria y Alemania.

El patrón más común que se reitera en los países de la UE es el permiso no transferible y, si lo es, se lleva a efecto sólo en circunstancias muy concretas, tales como enfermedad o fallecimiento de la madre. La posibilidad de poder negociar entre ambos progenitores quien solicita el permiso (a excepción de los días de obligatorio descanso para la madre), supone en la mayoría de los casos la selección del permiso por parte de la madre (OCDE, 2016c). En esta línea, consideramos interesante destacar que aquellos países que han introducido una serie de días de permiso exclusivamente para los padres sin que de ninguna forma se puedan transferir a las madres, están consiguiendo aumentar paulatinamente la elección de los padres de este tipo de permiso, como ya acontece en Suecia (OCDE, $2016 b)$. En este país, aquellas familias que comparten el permiso entre ambos progenitores reciben un bono diario, sin impuestos, de 50 coronas por un máximo de 270 días (Sverige, 2017). 
Seguidamente, reflejamos en la tabla 2 el pago por nacimiento en algunos países de la UE.

Tabla 2. Pago por nacimiento en algunos países de la UE.

\begin{tabular}{|c|c|}
\hline PAÍS & PAGO POR NACIMIENTO \\
\hline ALEMANIA & - \\
\hline AUSTRIA & - \\
\hline BÉLGICA & $1.247,58 €$ (primer hijo) y $938,66 €\left(2^{\circ}\right.$ y posteriores $)$. \\
\hline BULGARIA & 250 BGN (primer hijo); $600\left(2^{\circ}\right) ; 300 \mathrm{BGN}\left(3^{\circ}\right) ; 200 \mathrm{BGN}$ para cada uno de los hijos siguientes \\
\hline CHIPRE & 544,08 \\
\hline CROACIA & 2,328 \\
\hline DINAMARCA & - \\
\hline ESLOVAQUIA & $\begin{array}{l}829,86 €\left(1^{\circ}, 2^{\circ} \text { y } 3^{\circ} \text { hijo }\right) ; 151,37 € \text { por el } 4^{\circ} \text { hijo y por hijo adicional. } \\
\text { Por parto múltiple } 110,36 € \text { al año durante los dos primeros años. }\end{array}$ \\
\hline ESLOVENIA & Si el ingreso mensual per cápita es inferior al $64 \%$ del salario medio neto. Importe: $280 €$ \\
\hline ESPAÑA & Parto múltiple (2 o más niños). 2.620,80 € (2 niños); $5.241,60 €$ (3 niños) y 7.862,40 € (para 4 o más niños). \\
\hline ESTONIA & $320 €$ \\
\hline FINLANDIA & $140 €$ \\
\hline FRANCIA & $923,08 €$ \\
\hline GRECIA & - \\
\hline HUNGRÍA & 64,125 HUF para cada niño y 85.500 HUF si son gemelos \\
\hline IRLANDA & $10,16 €$ \\
\hline ITALIA & No hay subsidio por nacimiento, sólo para familias numerosas con cuatro o más hijos. Importe: $500 €$ \\
\hline LETONIA & $421,17 €$ por cada niño \\
\hline LITUANIA & $418 €$ \\
\hline LUXEMBURGO & $1.740,09 €$ \\
\hline MALTA & - \\
\hline P. BAJOS & - \\
\hline POLONIA & Si el ingreso neto familiar per cápita es inferior a 1922. La ayuda es de 1000 PLN \\
\hline PORTUGAL & - \\
\hline REINO UNIDO & 500 GBP \\
\hline R. CHECA & $829,86 €\left(1^{\circ}, 2^{\circ}\right.$ y $3^{\circ}$ hijo $) ; 151,37 €$ por el $4^{\circ}$ y por cada hijo adicional. \\
\hline RUMANÍA & - \\
\hline SUECIA & - \\
\hline
\end{tabular}

Fuente: OCDE (2017).

Como podemos observar en la tabla 2, de todos los Estados miembros, Luxemburgo tiene una de las prestaciones por nacimiento más elevadas, con 1.740,09€. Le sigue Bélgica con un total de 1.247,58€ por el primer hijo y 938,66€ por el segundo y posteriores. El país con una prestación por nacimiento más baja es Irlanda con 10,16€. Finalmente, cabe destacar que aún existen muchos países de la UE que no otorgan ningún tipo de ayuda por nacimiento, como son: Suecia, Rumanía, Portugal, Países Bajos, Malta, Grecia, Dinamarca, Austria y Alemania. 


\section{CONCLUSIONES}

Tras referirnos a las políticas de conciliación familiar-laboral que se llevan a cabo en los países de la UE, nos hemos centrado en los permisos parentales (teniendo en cuenta diversos aspectos) y en las ayudas por nacimiento que se conceden. Hemos podido comprobar la heterogeneidad de las políticas de apoyo a las familias que se están desarrollando en los distintos Estados miembros de la UE en función del modelo de bienestar seguido en cada país. Dichas políticas pretenden disminuir algunas de las dificultades económicas y laborales que se derivan fundamentalmente del nacimiento de los hijos. En líneas generales, a tal efecto se pretende realizar un reparto equitativo de las responsabilidades y prestar una mayor atención a determinadas situaciones familiares.

Uno de los retos que se afrontan para mejorar la conciliación familiar-laboral consiste en aumentar el número de padres que se acogen al permiso de paternidad. Se desea alcanzar una verdadera corresponsabilidad en el cuidado de los hijos y evitar cualquier tipo de perjuicio que pueda recaer sobre las madres relacionado con su derecho al empleo y a su desarrollo profesional.

Las políticas de apoyo a la familia son un requisito imprescidible para mejorar las condiciones de vida de la población y, especialmente, de la primera infancia, así como favorecer la igualdad de oportunidades, especialmente las relativas al acceso de la mujer al mercado laboral. No obstante, se ha de tener presente que las políticas familiares tienen tienen verdaderas dificultades para influir en las decisiones que se toman dentro del entorno familiar, tales como el reparto de tareas entre ambos cónyuges. Si bien, algunas medidas, como la de ofrecer unos adecuados incentivos por el cuidado de los hijos y la de reservar un período obligatorio de permiso para los padres, están demostrando tener buenos resultados, estimamos necesario que dichas medidas políticas vayan acompañadas de la realizacion de campañas de sensibilizacion y de un mayor énfasis en las políticas educativas dirigidas a las futuras generaciones (OCDE, 2016a).

\section{REFERENCIAS BIBLIOGRÁFICAS}

Davies, R. (2013). Work-life balance. Measures to help reconcile work, private and family life. Library Briefing. Recuperado de http:// www.europarl.europa.eu/RegData/bibliotheque/briefing/2013/130549/LDM_BRI\%282013\%29130549_REV1_EN.pdf.

Eurofound (2015). Promoting parental and paternity leave among fathers. Recuperado de https://www.eurofound.europa. eu/news/news-articles/working-conditions-industrial-relations/promoting-parental-and-paternity-leave-among-fathers-eurofound-news-march-2015.

European Parliament (2016). At a glance. Maternity and paternity leave in the UE. Recuperado de http://www.europarl.europa.eu/RegData/etudes/ATAG/2016/593543/EPRS_ATA(2016)593543_EN.pdf.

Eurostat (2017). Europe 2020 indicators-education. Recuperado de http://ec.europa.eu/eurostat/statistics-explained/index. php/Europe_2020_indicators_-_education.

Eurydice (2012). Cifras clave de la educación en Europa 2012. Comisión Europea.

González, J. (2014). El sesgo masculino y de género del derecho de la Unión Europea en torno a la conciliación de la vida familiar y laboral. Revista de Derecho de la Universidad César Vallejo, 51-56.

López, A., Acereda, A., Signes, M., y Amado , L. (2015). La conciliación laboral-familiar y su relación con el rendimiento escolar. Revista Internacional de Educación y Aprendizaje, 3 (1), 39-56.

McColgan, A. (2015). Measures to address the challenges of work-life balance in the EU Member States, Iceland, Liechtenstein and Norway. European Commission. Recuperado de: http://ec.europa.eu/justice/gender-equality/files/your_rights/11-1-2016_reconciliation_final.pdf.

OCDE. (2016a). LMF1.2: Maternal employment rates. Recuperado de http://www.oecd.org/els/family/LMF_1_2_Maternal_ Employment.pdf.

OCDE. (2016b). Parental leave: where are the fathers? Recuperado de https://www.oecd.org/policy-briefs/parental-leave-where-are-the-fathers.pdf.

OCDE. (2016c). PF2.4: Parental leave replacement rates. Recuperado de http://www.oecd.org/els/family/PF2_4_Parental_ leave_replacement_rates.pdf.

OCDE. (2017). PF2.1: Key characteristics of parental leave systems. Recuperado de http://www.oecd.org/els/soc/PF2_1_ Parental_leave_systems.pdf.

Sverige (2017). Gender Equality in Sweden. Recuperado de https://sweden.se/society/gender-equality-in-sweden/. 